\title{
Vitamin D, sunlight exposure, sleep disturbances and musculoskeletal health of older South Asian women in the UK: biological and social influences
}

\author{
A. L. Darling ${ }^{1}$, K. H. Hart ${ }^{1}$, D. J. Skene ${ }^{2}$, S. Arber ${ }^{3}$ and S. A. Lanham-New ${ }^{1}$ \\ ${ }^{1}$ Department of Nutritional Sciences, Faculty of Health and Medical Sciences, ${ }^{2}$ Department of Biochemistry and \\ Physiology, Faculty of Health and Medical Sciences, ${ }^{3}$ Department of Sociology, Faculty of Arts and Human Sciences, \\ University of Surrey, Guildford, GU2 7XH, UK
}

Older South Asian women in the UK are known to be at increased risk of vitamin D deficiency compared with their age-matched Caucasian counterparts ${ }^{(1)}$. There is an urgent need to better understand this problem, and its health effects. The first aim of this work was to assess musculoskeletal and sleep health in older South Asian and Caucasian women and to assess whether this is related to poorer vitamin D status. The second aim was to assess the social factors underpinning sun avoidance and poor vitamin D status in South Asian women.

As a follow up to the FSA-funded (2006-2010-N05064) D-FINES study (Vitamin D, Food Intake, Nutrition and Exposure to Sunlight

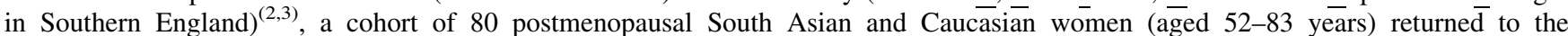
University of Surrey for further measurements of vitamin D status and musculoskeletal health and new measurements for light exposure and sleep-wake cycles. Bone structure was assessed by peripheral Quantitative Computed Tomography (pQCT) scans ( $n=68$ ). Muscle strength was assessed by stand to walk tests, grip strength, and a muscle and bone pain questionnaire $(n=80)$. A subset of subjects $(n=32)$ wore Actiwatches to monitor light exposure and actigraphic sleep-wake cycles. A subset of the older women $(60-70$ years) $(n=28)$ were interviewed about the social factors surrounding their sun exposure behaviour.

As expected, Asians had a significantly lower vitamin D concentration than Caucasians $(p=0.002)^{(3)}$. Indeed, $83 \%$ of Asians $v s .24 \%$ of Caucasians were below $50 \mathrm{nmol} / \mathrm{l}$ for 25 -hydroxyvitamin D $(25(\mathrm{OH}) \mathrm{D})$. Results from the pQCT scans suggested beneficial adaptations in bone structure of the tibia in Asians to improve bone strength. Asians, however, still had weaker bones by $38 \%$ compared with Caucasians $(p<0.001)$, due to their smaller bone size ${ }^{(4)}$. Self-reports of problems with tasks of daily living showed only marginally poorer function in the Asians than Caucasians (median score: Caucasians $=1 / 32$ vs. Asians $=3 / 32 ; p=0.008$ ). However, stand-to-walk time (Asian mean (SD) time $8.1 \mathrm{~s}(1.8) v s$. Caucasian mean (SD) time $6.9 \mathrm{~s}(1.4) ; p=0.002)$ and grip strength measures $($ Asian strength $=70 \%$ of Caucasian strength, $p<0.001)$ were worse in the Asians. However, there were no consistent associations between musculoskeletal health and vitamin D status in either group. In terms of sleep quality, Asians had lower actigraphic sleep efficiency $(p=0.003)$ and more fragmented sleep $(p=0.001)$ than Caucasians. Asian women also had less light exposure, with Asians spending less time per day in brighter light (over 500 lux) than Caucasians ( $p=0.03 ; n=14$ Caucasian and $n=5$ Asian). Qualitative analysis of the interview data suggested that religious and cultural influences on family, work and community life may partly explain the reduced sunlight exposure in South Asian women, thus contributing to vitamin D deficiency.

The implications of this work are that older South Asian women are in clear need of intervention to improve vitamin D status. There is also some evidence for poorer musculoskeletal health, lower light exposure and poorer sleep in this group. The qualitative research included in the current study offers future intervention options to improve vitamin D status, not least the importance of designing strategies to safely increase sunlight exposure in South Asians against the backdrop of their religious, family, work and community lives.

ALD is recipient of a Cross-Faculty University of Surrey $\mathrm{PhD}$ Scholarship.

1. Macdonald HM, Mavroeidi A, Fraser WD, Darling AL et al. (2012) Osteoporos Int 22 2461-2472.

2. Darling AL, Hart KH, Macdonald HM, et al. (2013) Osteoporos Int 24 477-488.

3. Darling AL, Hart KH, Gibbs MA, et al. (2013) Osteoporos Int Under $2^{\text {nd }}$ Review.

4. Darling AL, Hakim OA, Horton K, et al (2013) Bone in press. 